\title{
Sleep RR-Interval U-Patterns and Their Correlation to Movement Events
}

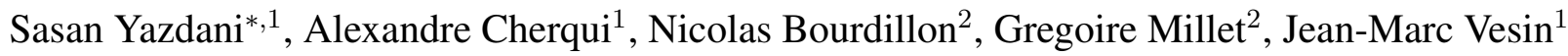 \\ ${ }^{1}$ Applied Signal Processing Group, Swiss Federal Institute of Technology, Lausanne, Switzerland \\ ${ }^{2}$ ISSUL, Institute of Sport Sciences, Faculty of Biology and Medicine, Unil, Lausanne, Switzerland
}

\begin{abstract}
The aim of this work is to investigate the relation between a phenomenon called "U-patterns" and their possible correlation to movement events in the context of sleep deprivation. U-patterns take place in the RR-interval time series during sleep. As their name suggests, these patterns present a U-shaped decrease-increase in RR-intervals, with a duration lasting from 20 to 40 seconds together with a minimum decrease of $15 \%$ in the local RR mean value.

Over a span of 17 days, 15 healthy subjects (7males, $22.1 \pm 1.7$ yrs.) participated in a study of three subsequent stages. First, a baseline phase of seven days, during which the subjects slept normally. Immediately after, a sleep deprivation phase with a duration of three days, during which participants slept only three hours per night. Finally, in a 7-day recovery phase subjects went back to their normal baseline sleeping routine. Subjects underwent polysomnography (PSG) data acquisition while sleeping. U-patterns were extracted from RR-intervals while movement events were extracted from different $P S G$ channels. Their relative temporal layout was studied to determine whether U-patterns are caused due to subject movement during sleep or vice versa. Results show that $U$ pattern/movement events are correlated, always initiated by U-patterns with movement events terminating before the termination of their respective $U$-patterns.
\end{abstract}

\section{Introduction}

Sleep analysis can reveal much information about human physiological health. A comprehensive sleep study can be performed through polysomnography (PSG) as it offers simultaneous recording of multiple biophysiological parameters. PSG records a number of bio-signals, from the electrical activity of the brain, i.e. electroencephalography (EEG), to eye movement tracking through electrooculography (EOG), nasal and abdominal respiration signals. Moreover, muscle and heart activity are monitored respectively via EMG and ECG recordings.

The benefits of sleep assessment have been pointed out in the literature, with researches showing the negative ef- fects of sleep deprivation on human health. Low quality of sleep and sleep deprivation have been linked to cognitive impairment [1], hypertension [2] and myocardial hypertrophy [3]. This highlights the importance of PSG analysis, which has already been deemed as an effective measure to identify disorders such as sleep apnea [4,5], insomnia [6], fatigue [7].

Even with the numerous studies carried out on sleep analysis, this interesting field remains one of the active research areas. The aim of this study is to investigate an intriguing phenomenon called "U-patterns", which manifest itself in the RR-interval time series during sleep. Upatterns were introduced and studied in [8] and [9], however the state-of-the-art had indirectly observed their existence [10]. This study aims to further investigate these patterns and analyze their correlation to movement events during sleep.

The remainder of this paper is organized as follows. Section 2 describes the research material used in this study. A short description of U-patterns, and the processing techniques to extract them as well as movement events are provided in section 3. Results are presented and discussed in Section 4. Finally in Section 5, the main conclusions of this work are drawn.

\section{Research Material}

The data used in this study were collected from 15 subjects (7 males and 8 females), whose anthropometric parameters are reported in Table 1.

This study was carried out over a span of 17 days, in three successive stages. First a "baseline" phase lasting for seven days, during which the subjects slept normally with no constraints. Following the baseline, subjects went through a "sleep deprivation" phase with a duration of

Table 1. Anthropometric parameters of this study.

\begin{tabular}{|c|c|c|}
\hline Characteristic & mean $\pm \operatorname{std}(N=15)$ & Range \\
\hline \hline Age $($ years $)$ & $22.1 \pm 1.7$ & $18-25$ \\
\hline Height $(\mathrm{cm})$ & $172.7 \pm 8.8$ & $160-196$ \\
\hline Weight $(\mathrm{kg})$ & $65.9 \pm 11.6$ & $52-92$ \\
\hline
\end{tabular}


three days, during which they could only sleep three hours at night. Finally, a 7-day "recovery" phase took place, during which subjects slept normally, as they would in the baseline phase. At the end of each phase, subjects underwent a night-long PSG recording.

\section{Methods}

\subsection{Definition of U-Patterns}

U-patterns are defined as U-shape decrease-increase in the RR-interval time series, with a duration of 20 to 40 seconds, and with a minimum decrease of $15 \%$ in the local RR-interval mean value, identical to the definition in [8]. By analyzing the RR-interval time series from night-long ECG recordings one can observe these patterns. Although recurrent within the RR-intervals, U-patterns do not seem to take place periodically or quasi-periodically. An example U-pattern is depicted in Figure 1, in which the extracted U-shaped pattern has a duration of 38.5 seconds with a decrease in mean RR-intervals of $29.6 \%$, from 927 ms down to $653 \mathrm{~ms}$.

\subsection{Extraction of U-Patterns and Move- ment Events}

ECG Preprocessing: Heartbeats were first extracted from night-long ECG recordings using a non-linear R-peak detector [11], which works on a filtering technique known as relative energy (Rel-En) and has proven to provide robust results in several applications [12-14]. The extracted RRinterval time series were first regularly resampled at $1 \mathrm{~Hz}$, and then smoothed using moving median filter with a duration of 15 seconds in order to remove small excursions.

U-pattern Extraction: The smoothed RR-intervals was used as input to the Rel-En algorithm [12] to extract Upattern candidates. The duration and depth of candidate patterns were then analyzed to further enhance U-pattern
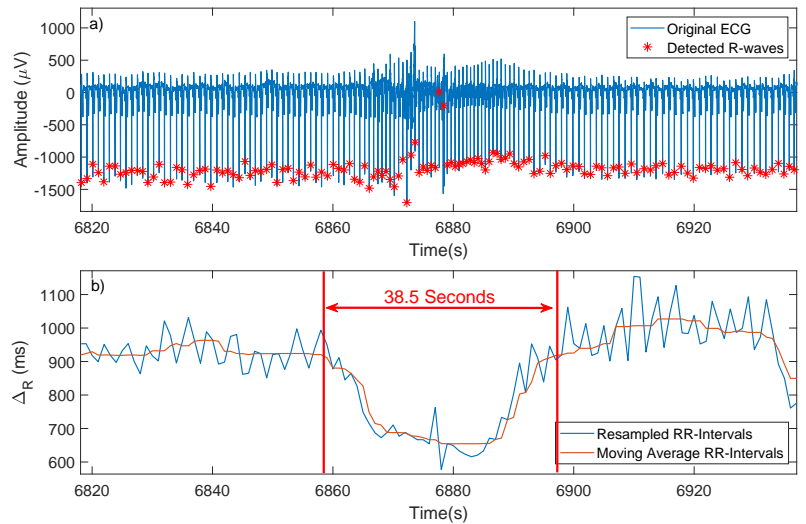

Figure 1. U-pattern taking place during sleep. a) The ECG excerpt containing the U-pattern alongside the detected Rwaves. b) The U-pattern in the RR-interval time series. detection (for details about the parameters and extraction technique see [8]).

Extraction of Movement Events: High-amplitude and frequency segments from different PSG channels were extracted as movement events. Observations on the available signals showed that the motor cortex EEG channels (C3-C4) along side the submentalis muscle EMG (3 leads) represented head movements to a higher degree, while the respiration signal (thorax channel) best represented body movements. Therefore, the aggregate set of movement events extracted from these channels (referred to as 3EMG) was used to assess U-pattern/movement event correlation.

In order to extract movement events each channel was processed by the Rel-En algorithm [12]. First, signals were normalized and Rel-En was applied using short- and longwindow durations of 20 and 2000 seconds, with an exponent of 2 as well as a Hamming window were used to enhance peak activities. Subsequently a hysteresis comparator were applied in order to extract movement events more robustly. Figure 2 illustrates the extracted movement events from the thorax channel of a night-long PSG recording.

\section{Results and Discussion}

Following the extraction of movement events, their correlation to U-patterns was studied. This task was carried out by comparing movement onset/offset times with those of the U-patterns. More specifically, a U-pattern was considered correlated with a movement event when there was an intersection between their respective time intervals, with a tolerance of five seconds. The agreement rate was then calculated as the percentage of U-patterns correlated with movement.

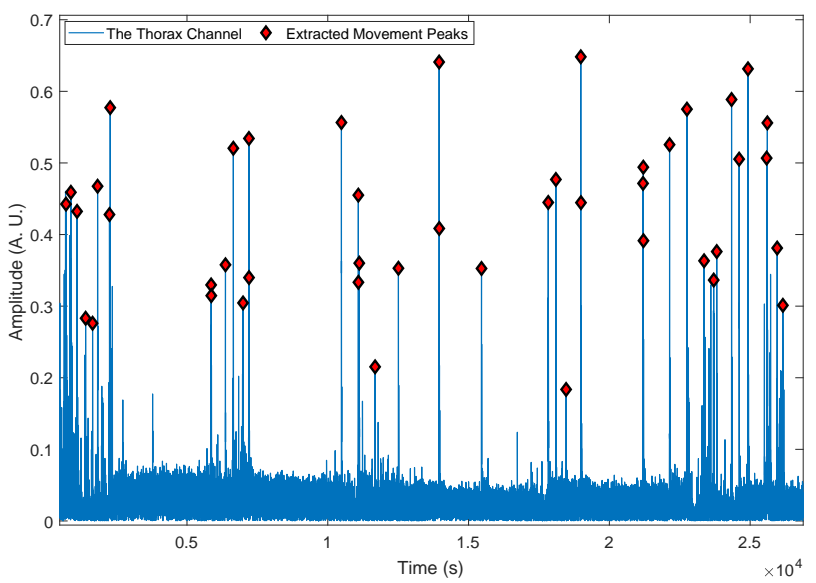

Figure 2. Illustration of night-long movement event extraction from the thorax channel. 


\begin{tabular}{|c|c|c|c|}
\hline Attribute & $\begin{array}{c}\text { Baseline } \\
(\text { mean } \pm \text { std })\end{array}$ & $\begin{array}{c}\text { Sleep Deprivation } \\
(\text { mean } \pm \text { std })\end{array}$ & $\begin{array}{c}\text { Recovery } \\
(\text { mean } \pm \text { std })\end{array}$ \\
\hline 3-EMG & $26.8 \pm 14.2$ & $26.3 \pm 13.6$ & $28.2 \pm 13.3$ \\
\hline Thorax & $39.9 \pm 16.0$ & $38.4 \pm 15.1$ & $43.0 \pm 8.4$ \\
\hline C3 & $24.7 \pm 13.3$ & $18.9 \pm 12.4$ & $24.9 \pm 12.6$ \\
\hline C4 & $24.9 \pm 12.9$ & $22.2 \pm 14.0$ & $23.7 \pm 10.5$ \\
\hline
\end{tabular}

Table 2. Inter-subject U-pattern/movement event agreement rate (\%) with respect to 3-EMG, thorax, $\mathrm{C} 3$ and $\mathrm{C} 4$ channels.

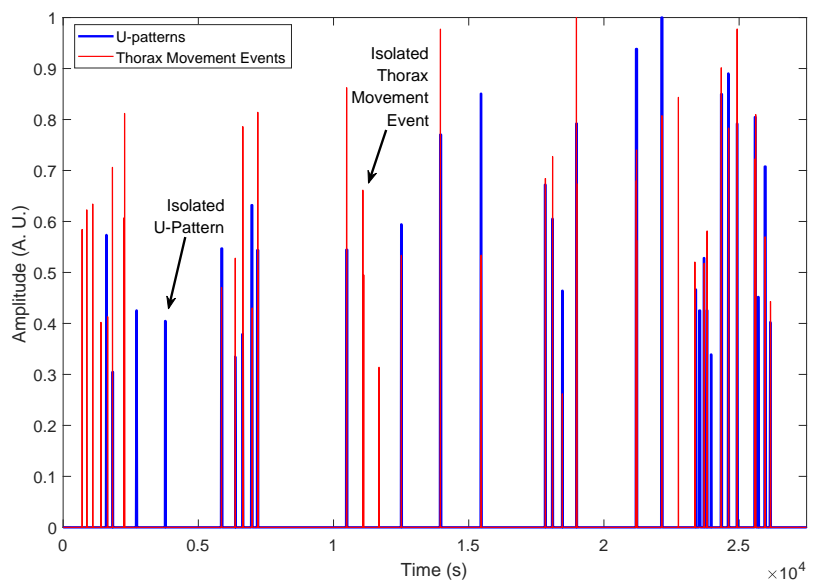

Figure 3. U-pattern/thorax movement event agreement for a night-long PSG recording from the sleep deprivation study, baseline phase.

Table 2 reports the U-pattern/movement agreement rate for each sleep deprivation phase. In this table, for each subject, U-patterns were compared with movement events from the $\mathrm{C} 3, \mathrm{C} 4$, thorax and 3-EMG channels (3-EMG represents the aggregation on the three available EMG leads, i.e. a 3-EMG movement event represents the extraction of a event from at least one of the three available EMG channels).

As reported in Table 2, movement events extracted from the thorax channel generally had the highest agreement rate with U-patterns. Furthermore, this was the case across all phases of the sleep deprivation study. Figure 3, illustrates an example U-pattern/movement agreement on a night-long PSG recording. In this figure, U-pattern amplitudes are normalized with respect to depth and duration of all subject U-patterns. Likewise, movement event amplitudes are normalized with respect to energy and duration of all subject movement events.

Results on U-pattern/movement agreement rates show that some U-patterns may not be correlated with any movement event. As shown in Figure 3, several U-patterns and movement events take place at the same time. However, some isolated U-patterns as well as isolated movement events can be observed.
Having extracted the correlated U-patterns/movement events, the causality between these two phenomena was studied. To this end, for correlated events, the onset time differences were calculated. Time delays of correlated events, for each sleep deprivation phase, are reported in Tables 3-4. Results show that correlated U-pattern/movement events are always initiated by U-patterns. Similarly, by studying U-pattern/movement offsets, the opposite results were obtained, i.e. U-patterns always terminated after their respective movement event.

Figure 4 displays an example of correlated Upattern/thorax movement event (same data as in Figure 3). One can observe that the U-pattern initiates the movement and disappears after the movement event is terminated. As U-patterns represent an increase in heart rate, one could explain this chain of events as follows:

1. A transient phase, during which the subject goes from deep to shallow sleep or a micro-arousal stage.

2. A movement phase, for instance change in posture from one side to another.

3. A second transient phase, during which the subject goes from shallow sleep or a micro-arousal stage back to deep sleep.

It should be mentioned that in this study, due to lack of relevant data, only head and thorax movement events were analyzed. In order to perform a more comprehensive movement analysis, one needs to take into consideration limb movements, i.e. hands and feet movement, as well. This is primarily due to the fact that these events might not be captured by head and trunk sensors, and therefore result in a lower overall U-pattern/movement agreement.

\begin{tabular}{|c|c|c|c|}
\hline Onset Delays (s) & $\begin{array}{c}\text { Baseline } \\
(\text { mean } \pm \text { std })\end{array}$ & $\begin{array}{c}\text { Sleep Deprivation } \\
(\text { mean } \pm \text { std })\end{array}$ & $\begin{array}{c}\text { Recovery } \\
(\text { mean } \pm \text { std })\end{array}$ \\
\hline 3-EMG & $8.9 \pm 1.5$ & $14.9 \pm 3.3$ & $8.0 \pm 2.2$ \\
\hline Thorax & $8.1 \pm 1.6$ & $13.2 \pm 3.1$ & $7.6 \pm 1.7$ \\
\hline C3 & $11.0 \pm 2.9$ & $14.6 \pm 2.8$ & $11.4 \pm 5.0$ \\
\hline C4 & $9.4 \pm 3.2$ & $15.9 \pm 3.2$ & $10.5 \pm 4.3$ \\
\hline
\end{tabular}

Table 3. Inter-subject averages of U-pattern/Movement onset delays (seconds), with respect to 3-EMG, thorax, C3 and $\mathrm{C} 4$ channels.

\begin{tabular}{|c|c|c|c|}
\hline Offset Delays (s) & $\begin{array}{c}\text { Baseline } \\
(\text { mean } \pm \text { std) }\end{array}$ & $\begin{array}{c}\text { Sleep Deprivation } \\
(\text { mean } \pm \text { std })\end{array}$ & $\begin{array}{c}\text { Recovery } \\
(\text { mean } \pm \text { std })\end{array}$ \\
\hline 3-EMG & $15.9 \pm 4.7$ & $8.5 \pm 2.0$ & $14.7 \pm 3.1$ \\
\hline Thorax & $13.1 \pm 2.3$ & $7.9 \pm 1.8$ & $12.9 \pm 2.2$ \\
\hline C3 & $13.8 \pm 5.3$ & $10.7 \pm 3.3$ & $14.2 \pm 3.5$ \\
\hline C4 & $14.4 \pm 4.3$ & $9.9 \pm 1.8$ & $14.2 \pm 3.5$ \\
\hline
\end{tabular}

Table 4. Inter-subject averages of U-pattern/Movement offset delays (seconds), with respect to 3-EMG, thorax, C3 and $\mathrm{C} 4$ channels. 


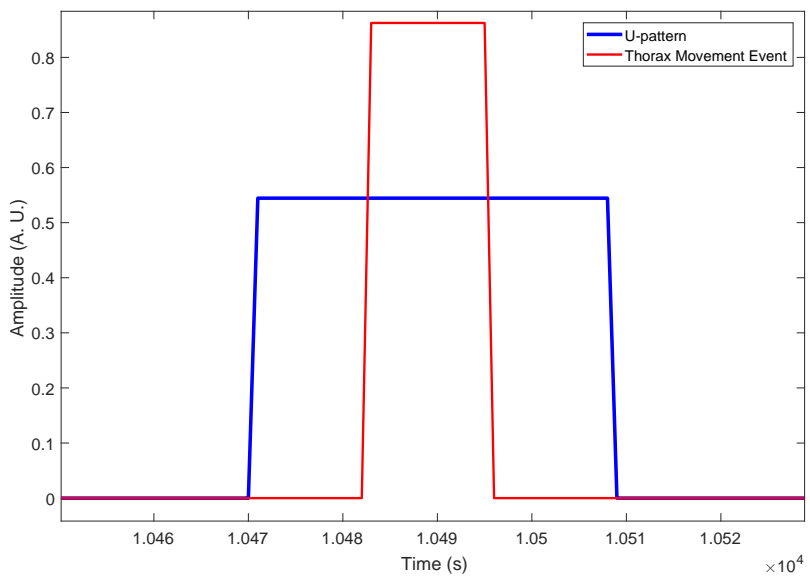

Figure 4. Causality between U-patterns/movement events. The U-pattern is initiated first, then movement event takes place, after which the U-pattern is terminated.

\section{Conclusion}

This paper studies a U-shape acceleration/deceleration phenomenon that is discernible in the RR-intervals of night-long PSG recordings. More specifically, the correlation between U-patterns and movement events during sleep is studied. Results suggest a moderate correlation between U-patterns and movement events. Furthermore, for correlated events investigations showed that U-patterns are always initiated before their corresponding movement events and terminated after a few seconds after movement.

\section{Acknowledgments}

This study was performed in the framework of the NanoTera initiative supported by the Swiss National Science Foundation (SNSF).

This work was made possible by the financial support of the CTI commission $\mathrm{n}^{\circ} 18164.2515$ PFLS-LS.

\section{References}

[1] Yaffe K, Laffan AM, Harrison SL, Redline S, Spira AP, Ensrud KE, Ancoli-Israel S, Stone KL. Sleep-disordered breathing, hypoxia, and risk of mild cognitive impairment and dementia in older women. Jama 2011;306(6):613-619.

[2] Sulit L, Storfer-Isser A, Kirchner HL, Redline S. Differences in polysomnography predictors for hypertension and impaired glucose tolerance. Sleep 2006;29(6):777-783.

[3] Fernandez-Mendoza J, Vgontzas AN, Liao D, Shaffer ML, Vela-Bueno A, Basta M, Bixler EO. Insomnia with objec- tive short sleep duration and incident hypertension. Hypertension 2012;108-112.

[4] Fujita S, Conway W, Zorick F, Roth T. Surgical correction of anatomic abnormalities in obstructive sleep apnea syndrome: uvulopalatopharyngoplasty. Otolaryngology Head and Neck Surgery 1981;89(6):923-934.

[5] Marcus CL, Chapman D, Ward SD, McColley SA, Herrerias CT, Stillwell PC, Howenstine M, Light MJ, Schaeffer DA, Wagener JS, et al. Clinical practice guideline: diagnosis and management of childhood obstructive sleep apnea syndrome. Pediatrics 2002;109(4):704-712.

[6] Bastien CH, Vallières A, Morin CM. Validation of the insomnia severity index as an outcome measure for insomnia research. Sleep medicine 2001;2(4):297-307.

[7] Krupp LB, Jandorf L, Coyle P, Mendelson W. Sleep disturbance in chronic fatigue syndrome. Journal of Psychosomatic Research 1993;37(4):325-331.

[8] Yazdani S, Cherqui A, Bourdillon N, Millet GP, Vesin JM. Analysis of U-shape patterns in RR-interval time series during sleep. In 2018 Computing in Cardiology Conference (CinC), volume 45. Sep. 2018; 1-4.

[9] Solinski M, Gieraltowski J, Zebrowski J, Kuklik P. Influence of $\mathrm{u}$-shape accelerations of heart rate on very low frequency band and heart rate multifractality. In 2017 Computing in Cardiology Conference (CinC). September 2017; 721-724.

[10] Dorantes-Méndez G, Mendez MO, Alba A, Parrino L, Milioli G. Time-varying analysis of the heart rate variability during a-phases of sleep: Healthy and pathologic conditions. Biomedical Signal Processing and Control 2018; 40:111-116.

[11] Yazdani S, Vesin JM. A novel preprocessing tool to enhance ECG R-wave extraction. In The 43rd annual international conference of Computing in Cardiology. September 2016; 633-636.

[12] Yazdani S, Fallet S, Vesin JM. A novel short-term event extraction algorithm for biomedical signals. IEEE Transactions on Biomedical Engineering April 2018;65(4):754762. ISSN 0018-9294.

[13] Vesin JM, Yazdani S, Mirmohamadsadeghi L, Bourdillon N. Extraction and analysis of short-time excursions in RRinterval time series. In 2016 Computing in Cardiology Conference (CinC). September 2016; 721-724.

[14] Orlandic L, De Giovanni E, Arza Valdes A, Yazdani S, Vesin JM, Atienza Alonso D. Reward: Design, optimization, and evaluation of a real-time relative-energy wearable R-peak detection algorithm. In Engineering in Medicine and Biology Conference (EMBC). July 2019; 1-1.

Address for correspondence:

Sasan Yazdani

EPFL SCI STI JMV - ELE 121 - Station 11

CH-1015 Lausanne - Switzerland.

E-mail address: sasan.yazdani@epfl.ch 